\title{
Análisis Comparativo de la Eficacia, Comodidad y Seguridad del Cepillo Eléctrico Oscilante-Rotatorio Oral-B en Pacientes Portadores de Ortodoncia Fija: Un Ensayo Clínico Aleatorizado
}

\author{
Comparative Analysis of the Efficacy, Comfort and Safety of the Oral-B Oscillating-Rotary \\ Electric Toothbrush in Patients with Fixed Orthodontia: A Randomized Clinical Trial
}

Pamela Araya-Díaz'; Natasha Abdala Torres²; Karim Yagnam Díaz; Hernán Palomino M.'; Juan Guillermo Parada Ibañez'; Eduardo Pastén Castro ${ }^{1}$ \& Soledad Piñeiro Becerra ${ }^{1}$

ARAYA-DÍAZ, P.; ABDALA, T. N.; YAGNAM, D. K.; PALOMINO, M. H.; PARADA, I. J. G.; PASTÉN, C. E. \& PIÑEIRO, B. S. Análisis comparativo de la eficacia, comodidad y seguridad del cepillo eléctrico oscilante-rotatorio Oral-B en pacientes portadores de ortodoncia fija: Un ensayo clínico aleatorizado. Int. J. Odontostomat. 15(3):765-772, 2021.

RESUMEN: La higiene oral es fundamental en pacientes en tratamiento de ortodoncia. Actualmente hay una oferta amplia de cepillos eléctricos con este fin. El objetivo del presente estudio, fue determinar la eficacia y la comodidad del cepillo eléctrico Oral-B® Professional Care 500 en pacientes portadores de ortodoncia. Se realizó un ensayo clínico, randomizado, doble ciego, unicéntrico, con una muestra de 90 pacientes, divididos en dos grupos: 45 pacientes utilizaron cepillo eléctrico y 45 sujetos utilizaron cepillos manuales durante 90 días. Se determinó la efectividad con los índices O'leary, índice de placa de bracket e índice de Eastman al inicio, a los 30 y a los 90 días de uso de los cepillos y se evaluó la comodidad del cepillo eléctrico mediante una encuesta de satisfacción al término del estudio. Al analizar los datos, se observó una disminución significativa en los índices de O'leary y de placa de bracket en ambos grupos,mientras que se observó una disminución significativa en el índice de Eastman sólo en el grupo que utilizó el cepillo eléctrico. El resultado de la encuesta, indica que, en opinión de los participantes del estudio, el cepillo eléctrico es cómodo, fácil de usar y recomendable para pacientes con aparatología fija. En conclusión, el cepillo eléctrico Oral-B® Professional Care 500 resultó ser efectivo y cómodo al ser usado por pacientes portadores de ortodoncia fija y puede ser recomendado para este tipo de pacientes.

PALABRAS CLAVE: ortodoncia, cepillado de dientes, salud oral, comodidad del paciente, índice de placadental.

\section{INTRODUCCIÓN}

Mantener una adecuada higiene, es uno de los objetivos fundamentales para mantener la salud oral en los pacientes portadores de ortodoncia. Se ha observado que el $60 \%$ de los pacientes ortodóncicos, presentan una alteración en la acumulación del biofilm inmediatamente después de la instalación de los aparatos de ortodoncia, aumentando especies del complejo amarillo (Veillonella parvula y Neisseria mucosa) y púrpura (Streptococcus) además de especies de cándida y pseudomonas que no están presentes en individuos sanos (Sun et al., 2018; Bergamo et al., 2019).

La falta de control de higiene dental se ha asociado a inflamación y agrandamiento gingival, pérdida de inserción, disminución de los tejidos de soporte y reabsorción radicular (Dersot, 2010). También se ha reportado la formación de manchas blancas en relación al área gingival en dientes con brackets al comenzar el tratamiento, describiéndose su evolución en

\footnotetext{
${ }^{1}$ Area de Ortodoncia, Facultad de Odontología, Universidad Andres Bello, Santiago, Chile.

${ }^{2}$ Alumna de Postgrado de Ortodoncia, Facultad de Odontología, Universidad Andres Bello, Santiago, Chile.

${ }^{3}$ Alumno de Postgrado de Ortodoncia, Facultad de Odontología, Universidad de Chile, Santiago, Chile.
} 
ARAYA-DÍAZ, P.; ABDALA, T. N.; YAGNAM, D. K.; PALOMINO, M. H.; PARADA, I. J. G.; PASTÉN, C. E. \& PIÑEIRO, B. S. Análisis comparativo de la eficacia, comodidad y seguridad del cepillo eléctrico oscilante-rotatorio Oral-B en pacientes portadores de ortodoncia fija: Un ensayo clínico aleatorizado. Int. J. Odontostomat. 15(3):765-772, 2021.

un mes en comparación a los seis meses que demora en desarrollarse en un diente libre de brackets (Khoroushi \& Kachuie, 2017).

Existen medidas coadyuvantes para controlar el biofilm y evitar la gingivitis como: pastas, enjuagues y barnices fluorados, probióticos, polioles, CPP-ACP (cadein phosphopeptides-amorphouse calcium phosphate) y antisépticos como la clorhexidina (Khoroushi \& Kachuie). Sin embargo, la literatura sigue soportando que la remoción de placa bacteriana de forma mecánica es el método más efectivo para su control.

En Chile, se ha recetado tradicionalmente a los pacientes con ortodoncia fija, el uso de cepillos manuales de ortodoncia. Actualmente, la oferta de cepillos eléctricos dentales ha ido en aumento como alternativa práctica, al resumir varias funciones en un solo cepillo, pudiendo aparecer como un elemento innovador y motivador a largo plazo en el tratamiento ortodóncico (Heintze et al., 1996). Su eficacia ha sido demostrada en varios estudios, entre ellos, una revisión de Cochrane de 2014, donde se determinó mejores resultados con cepillos eléctricos rotativos y oscilantes, en comparación a los manuales para la reducción de la inflamación gingival y el índice de placa a largo plazo (Yaacob et al., 2014). En Chile, se encuentra disponible hace un tiempo, el cepillo eléctrico Oral$B \circledR$ Professional Care 500, un cepillo accesible económicamente y que podría ser usado como alternativa a los cepillos manuales que tradicionalmente se indican en pacientes portadores de aparatología fija. Sin embargo, existe escasa evidencia científica disponible que permita determinar si este cepillo en específico es efectivo en eliminar la placa bacteriana en pacientes ortodóncicos.

El objetivo de este estudio es determinar la eficacia en el control de placa bacteriana y la percepción de los usuarios respecto de la comodidad y facilidad de uso del cepillo eléctrico Oral-B® Professional Care 500 , en pacientes portadores de ortodoncia fija.

\section{MATERIAL Y MÉTODO}

El estudio corresponde a un ensayo clínico randomizado, doble ciego, unicéntrico, el cual obtuvo la aprobación del comité de Ética institucional de la Universidad Andrés Bello para su desarrollo durante el año 2019.
El universo contemplado, correspondió a pacientes atendidos en el Programa de Especialización en Ortodoncia y Ortopedia Dentomaxilofacial de la Universidad Andrés Bello portadores de aparatología fija multibrackets, del cual se obtuvo una muestra que cumpliera con los siguientes criterios:

- Criterios de inclusión: pacientes entre 15-30 años, dentición permanente completa (sin considerar exodoncias por indicación ortodóncica), asistencia regular a controles desde hace al menos 6 meses, y que voluntariamente accedieran a participar del estudio firmando el consentimiento informado.

- Criterios de exclusión: pacientes en tratamiento con medicamentos que alteren la condición periodontal, portadores de síndromes o malformaciones cráneofaciales, con alteraciones cognitivas o con psicomotricidad disminuida, con historial de rotura o descementado de los aparatos, mujeres embarazadas o en período de lactancia.

- Se calculó el tamaño muestral considerando un $\alpha: 0,05$, un $\beta: 0,05$, una diferencia esperada de 15 y una varianza de 256 .Se ajustó a un $15 \%$ de pérdidas, según lo cual, se requerían un mínimo de 35 participantes por grupo, sin embargo, se logró incorporar 90 pacientes que cumplían con los criterios y accedieron a formar parte del estudio, por lo que cada grupo estaría conformado por 45 pacientes.

Se tabularon los datos estadísticos de los 90 participantes, y se realizó un muestreo probabilístico aleatorio simple mediante la función de aleatorización de Excel. De esta manera quedaron conformados los dos grupos:

Grupo experimental: 45 pacientes a los cuales, un investigador que no participaría posteriormente en las mediciones les entregó e instruyó en el uso del cepillo eléctrico oscilante-rotatorio de Oral-B(C 500.

Grupo control: 45 pacientes a los cuales, un investigador que no participaría posteriormente en las mediciones, se les instruyó en el uso de los cepillos Monotip y Orthodontic PHB de DENTAIDC.

Las instrucciones entregadas verbalmente y por escrito, contemplaban el uso de pasta dental Oral-B para ambos grupos; técnica horizontal en la zona de los brackets; técnica de Bass adaptada en las superficies libres para los portadores de cepillo manual y la técnica recomendada por Oral-B para el uso del cepillo eléctrico. En ambos casos se indicó una duración mínima de cepillado de dos minutos y se señaló evitar el uso de seda dental, enjuagues bucales o cualquier otro elemento que complemente la higiene oral. 
ARAYA-DÍAZ, P.; ABDALA, T. N.; YAGNAM, D. K.; PALOMINO, M. H.; PARADA, I. J. G.; PASTÉN, C. E. \& PIÑEIRO, B. S. Análisis comparativo de la eficacia, comodidad y seguridad del cepillo eléctrico oscilante-rotatorio Oral-B en pacientes portadores de ortodoncia fija: Un ensayo clínico aleatorizado. Int. J. Odontostomat. 15(3):765-772, 2021.

Para la evaluación clínica, se designaron dos examinadores ciegos, los cuales se sometieron a un proceso de calibración intra e interexaminador, bajo la evaluación de un especialista en Periodoncia. Los pacientes fueron examinados en 3 períodos de tiempo: inicial (T0), a los 30 días (T1) y a los 90 días(T2), en los cuales se evaluaron:

\section{Eficacia}

Mediante 3 índices. a) Índice de placa modificado O'Leary: Se evalúa la superficie dentaria visualmente y luego con sonda de caries para poder puntuar: 0 ausencia de placa bacteriana, 1 para presencia de placa bacteriana; b) Índice de placa en bracket: Se aplica una solución reveladora y se evalúa las superficies teñidas alrededor del bracket (mesial, distal, gingival, incisal y bracket). Se puntúa 0 para ausencia de placa, 1 para islotes aislados de placa bacteriana, 2 para área de placa menor a $1 \mathrm{~mm}$ y 3 para área de placa mayor a $1 \mathrm{~mm}$. c) Índice de sangrado gingival Eastman: Consiste en la inserción de un palillo de madera estéril en los espacios interdentarios mesial y distal de cada pieza examinada, presionando 1 a $2 \mathrm{~mm}$. la papila vestibular, se esperan 15 segundos y se registra 1 para sangrado y 0 para no sangrado. Los sitios con sangrado se representan en porcentaje.

Una vez obtenidos los registros iniciales, se tabularon los datos en formato Excel y se analizaron mediante estadística descriptiva e inferencial.

La estadística descriptiva consistió en la obtención de medias, desviaciones estándar, medianas y frecuencias de cada variable.

Para el análisis inferencial, se determinaron en primer lugar la distribución de las variables (test Shapiro-Wilks) y se realizaron las siguientes comparaciones:
- Comparación de índices en T0, T1 y T2 mediante el cálculo del análisis de varianza de Friedman + Post-Hoc tanto en el grupo experimental como en el grupo control. - Comparación grupo experimental v/s grupo control, tanto en T0 como T1 y T2, mediante el cálculo, según distribución, del test $t$ para muestras independientes o el test de Mann-Whitney U.

- Comparación de las diferencias aritméticas T2-T0, entre el grupo experimental y el grupo control mediante el test $t$ para muestras independientes o el test de Mann-Whitney U, según distribución.

Todos los análisis se realizaron con un nivel de confianza del $95 \%$, utilizando el software estadístico SPSS 25.0 para macOS.

Percepción de comodidad y facilidad de uso: Mediante una encuesta de satisfacción inicial y al finalizar el estudio, se evaluó la percepción de los pacientes del grupo experimental respecto del cepillo eléctrico.

La encuesta fue validada mediante el método Delphi, con un grupo de expertos constituido por 9 participantes. Los valores obtenidos en relación con la competencia de los expertos que participaron de la validación, se muestran en la Tabla I.

Dada las opiniones de los expertos, se realizaron modificaciones y se eliminaron preguntas poco pertinentes, hasta lograr el acuerdo de todos los consultados. Finalmente, la encuesta estuvo conformada por 16 preguntas relacionadas con aceptación, comodidad y facilidad de uso del cepillo. Los datos obtenidos de la encuesta fueron analizadas con estadística descriptiva.

\section{RESULTADOS}

Muestra: Durante el desarrollo del estudio, 12 pacientes no asistieron a los 3 controles (T0, T1 y T2), por lo

Tabla I. Competencia de los profesionales que formaron parte del panel de expertos para la validación de la encuesta según el método Delphi.

\begin{tabular}{cccc}
\hline $\begin{array}{c}\text { Co eficiente de } \\
\text { conocimiento }(\mathrm{Kc})\end{array}$ & $\begin{array}{c}\text { Co eficiente de } \\
\text { argumentación }(\mathrm{Ka})\end{array}$ & $\begin{array}{c}\text { Coeficiente de } \\
\text { competencia }(\mathrm{K})\end{array}$ & Valoración \\
\hline 0,8 & 0,8 & 0,8 & Alto \\
1 & 1 & 1 & Alto \\
0,8 & 0,8 & 0,8 & Alto \\
0,8 & 0,8 & 0,8 & Alto \\
0,8 & 0,8 & 0,8 & Alto \\
0,7 & 0,8 & 0,75 & Me dio \\
0,8 & 0,8 & 0,8 & Alto \\
0,8 & 0,8 & 0,8 & Alto \\
1 & 0,8 & 0,9 & Alto \\
\hline
\end{tabular}


ARAYA-DÍAZ, P.; ABDALA, T. N.; YAGNAM, D. K.; PALOMINO, M. H.; PARADA, I. J. G.; PASTÉN, C. E. \& PIÑEIRO, B. S. Análisis comparativo de la eficacia, comodidad y seguridad del cepillo eléctrico oscilante-rotatorio Oral-B en pacientes portadores de ortodoncia fija: Un ensayo clínico aleatorizado.Int. J. Odontostomat. 15(3):765-772, 2021.

Tabla II. Características epidemiológicas de la muestra final.

\begin{tabular}{lccccccccc}
\hline Ce pillos & \multicolumn{4}{c}{ Hombre } & \multicolumn{2}{c}{ Mujer } & \multicolumn{3}{c}{ Total } \\
& $\mathrm{n}$ & X edad & DS & $\mathrm{n}$ & X edad & DS & $\mathrm{n}$ & X edad & DS \\
\hline Eléctrico & 19 & 22,5 & 7 & 23 & 20,9 & 4,8 & 42 & 21,6 & 5,9 \\
Manual & 10 & 24,6 & 4,8 & 26 & 24,4 & 5,2 & 36 & 24,5 & 5,3 \\
\hline
\end{tabular}

cual fueron eliminados de la muestra. De esta manera, la muestra quedó constituida como se señala en la Tabla II.

Al comparar la situación inicial de ambos grupo, no se encontró diferencia estadísticamente significativa en ninguno de los índices evaluados (O'Learyp: 0,297 ; Placa en Bracket p:0,115 ; Eastman p:2,958) .

\section{Eficacia}

a) Índice de placa modificado O'Leary: Al comparar entre T0, T1 y T2, se observó una disminución estadísticamente significativa $(<0,05)$ en el índice de O'Leary, tanto con el uso del cepillo eléctrico, como con el uso de cepillos manuales.

Al comparar los resultados entre ambos cepillos, no se observó una diferencia estadísticamente significativa entre las variables analizadas (Tabla III). b) Índice de placa en bracket: Al comparar entre T0, T1 y T2, se observó una disminución estadísticamente significativa $(<0,05)$ en el índice de placa en bracket, tanto con el uso del cepillo eléctrico, como con el uso de cepillos manuales.

Al comparar los resultados entre ambos cepillos, se observó que existe diferencia estadísticamente significativa en la magnitud de la disminución observada en el índice de placa en bracket a los 30 días, siendo mayor la disminución en pacientes que utilizaron el cepillo eléctrico (Tabla IV).

c) Índice de sangrado gingival Eastman: Al comparar entre T0, T1 y T2, se observó una disminución estadísticamente significativa $(<0,05)$ en el índice de Eastman, sólo con el uso del cepillo eléctrico.

Al analizar la diferencia observada en el índice de Eastman en los distintos tiempo de examen (T0-T1

Tabla III. Resultados y análisis estadísticos del índice de placa modificado de O’leary.

\begin{tabular}{|c|c|c|c|c|c|c|c|c|}
\hline Período & Cepillos & $\mathrm{n}$ & $\overline{\bar{x}}$ & $\overline{D S}$ & Me diana & $\begin{array}{l}\text { Sig. } \\
\text { Test t }\end{array}$ & $\begin{array}{l}\text { Sig. U Mann- } \\
\text { Whitney }\end{array}$ & $\begin{array}{c}\text { Sig. } \\
\text { Fried man }\end{array}$ \\
\hline T0 & $\begin{array}{l}\text { Eléctrico } \\
\text { Manual }\end{array}$ & $\begin{array}{l}42 \\
36\end{array}$ & $\begin{array}{l}63,3 \\
59,3\end{array}$ & $\begin{array}{l}15,9 \\
17,6\end{array}$ & $\begin{array}{c}63,5 \\
60\end{array}$ & 0,297 & & $\begin{array}{c}\text { Eléctrico } \\
0,011^{*}\end{array}$ \\
\hline T1 & $\begin{array}{l}\text { Eléctrico } \\
\text { Manual }\end{array}$ & $\begin{array}{l}42 \\
36\end{array}$ & $\begin{array}{l}56,6 \\
48,4\end{array}$ & $\begin{array}{l}16,4 \\
17,2\end{array}$ & $\begin{array}{l}59,5 \\
48,5\end{array}$ & & 0,155 & $\begin{array}{c}\text { Manual } \\
0,000^{*}\end{array}$ \\
\hline T2 & $\begin{array}{l}\text { Eléctrico } \\
\text { Manual }\end{array}$ & $\begin{array}{l}42 \\
36\end{array}$ & $\begin{array}{l}54,4 \\
46,7\end{array}$ & $\begin{array}{l}17,7 \\
18,9\end{array}$ & $\begin{array}{c}55 \\
46,5\end{array}$ & 0,068 & & \\
\hline T0-T1 & $\begin{array}{l}\text { Eléctrico } \\
\text { Manual }\end{array}$ & $\begin{array}{l}42 \\
36\end{array}$ & $\begin{array}{l}-6,69 \\
-10,9\end{array}$ & $\begin{array}{l}17,8 \\
12,4\end{array}$ & $\begin{array}{c}-6 \\
-12\end{array}$ & 0,233 & & \\
\hline T0-T2 & $\begin{array}{l}\text { Eléctrico } \\
\text { Manual }\end{array}$ & $\begin{array}{l}42 \\
36\end{array}$ & $\begin{array}{l}-8,88 \\
-12,5\end{array}$ & $\begin{array}{l}22,1 \\
13,8\end{array}$ & $\begin{array}{c}-10,5 \\
-11\end{array}$ & 0,373 & & \\
\hline
\end{tabular}

Tabla IV. Resultados y análisis estadísticos del índice de placa en bracket.

\begin{tabular}{llcccccr}
\hline Período & Cepillos & $\mathrm{n}$ & $\bar{X}$ & DS & Mediana & Sig. Test t & Sig. Friedman \\
\hline T0 & Eléctrico & 42 & 2,2 & 0,45 & 2,26 & 0,115 & Eléctrico $0,000^{*}$ \\
& Manual & 36 & 2,02 & 0,55 & 2,07 & & Manual $0,000^{*}$ \\
T1 & Eléctrico & 42 & 1,69 & 0,64 & 1,79 & 0,626 & \\
& Manual & 36 & 1,62 & 0,66 & 1,57 & & \\
T2 & Eléctrico & 42 & 1,46 & 0,63 & 1,42 & 0,295 & \\
\multirow{2}{*}{ T0-T1 } & Manual & 36 & 1,31 & 0,59 & 1,28 & & \\
& Eléctrico & 42 & $-0,50$ & 0,70 & $-0,75$ & $0,047^{*}$ & \\
T0-T2 & Manual & 36 & $-0,39$ & 0,64 & $-0,32$ & & \\
& Eléctrico & 42 & $-0,74$ & 0,76 & $-0,75$ & 0,806 & \\
& Manual & 36 & -0.7 & 0,47 & $-0,69$ & & \\
\hline
\end{tabular}


ARAYA-DÍAZ, P.; ABDALA, T. N.; YAGNAM, D. K.; PALOMINO, M. H.; PARADA, I. J. G.; PASTÉN, C. E. \& PIÑEIRO, B. S. Análisis comparativo de la eficacia, comodidad y seguridad del cepillo eléctrico oscilante-rotatorio Oral-B en pacientes portadores de ortodoncia fija: Un ensayo clínico aleatorizado. Int. J. Odontostomat. 15(3):765-772, 2021.

Tabla V. Resultados y análisis estadísticos del índice de Eastman.

\begin{tabular}{lllccccc}
\hline Período & Cepillos & $\mathrm{n}$ & $\bar{x}$ & DS & Mediana & $\begin{array}{c}\text { Sig. U Mann- } \\
\text { Withney }\end{array}$ \\
\hline T0 & Eléctrico & 42 & 12,64 & 13,93 & 7 & 2,958 & Eléctrico \\
& Manual & 36 & 5,27 & 7,53 & 0 & & $0,007^{*}$ \\
T1 & Eléctrico & 42 & 7,23 & 11,25 & 2,5 & 1,455 & Manual \\
& Manual & 36 & 4 & 7,75 & 0 & & 0,121 \\
T2 & Eléctrico & 42 & 5,3 & 7,49 & 0 & 1,287 & \\
& Manual & 36 & 3,16 & 7,24 & 0 & & \\
T0-T1 & Eléctrico & 42 & $-5,4$ & 11,8 & 0 & 0,063 & \\
T0-T2 & Manual & 36 & $-1,2$ & 7,1 & 0 & & \\
& Eléctrico & 42 & $-7,33$ & 14,35 & 0 & 0,173 & \\
& Manual & 36 & $-2,1$ & 7,3 & 0 & &
\end{tabular}

y T2), se observa una disminución tres veces mayor del porcentaje de sitios con sangrado con el uso de cepillo eléctrico, sin embargo, la diferencia no resulta ser estadísticamente significativa (Tabla V).

Percepción de comodidad y facilidad de uso del cepillo eléctrico: De las respuestas entregadas, al finalizar el estudio, por los 42 pacientes a los cuales se les entregó el cepillo Oral-B® Professional Care 500 , se destacan las siguientes:

En relación con la pregunta: "¿Cómo calificaría la comodidad de uso del cepillo eléctrico entregado (Ausencia de molestias al realizar el cepillado)?", los resultados obtenidos se señalan en el siguiente gráfico (Fig. 1).

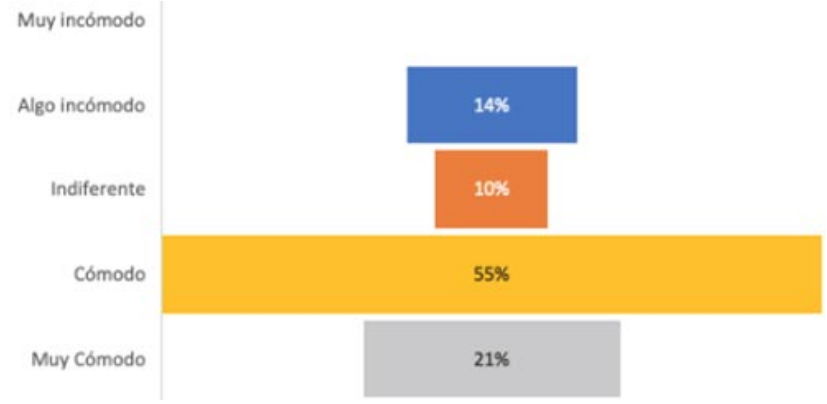

Fig. 1. Porcentajes de respuestas obtenidas en la encuesta en relación con la comodidad el cepillo eléctrico.

Frente a la pregunta: "Comparando la comodidad de uso del cepillo eléctrico entregado con los cepillos manuales que ha utilizado anteriormente, podría señalar que el cepillo eléctrico es". El $76 \%$ de los encuestados señaló que el cepillo eléctrico es más cómodo o mucho más cómodo que los cepillos manuales (más cómodo $55 \%$, mucho más cómodo $21 \%$ ).
En relación con la pregunta: "¿Cómo calificaría la facilidad de uso del cepillo eléctrico entregado (Ausencia de molestias al realizar el cepillado)?", los resultados obtenidos se señalan en el siguiente gráfico (Fig. 2).

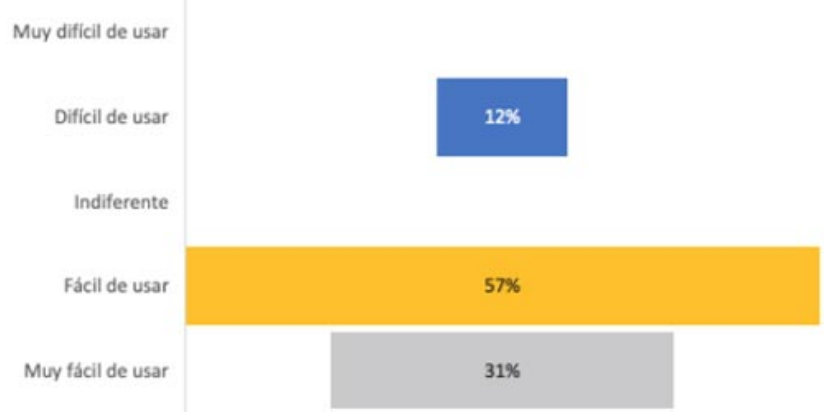

Fig. 2. Porcentajes de respuestas obtenidas en la encuesta en relación a la facilidad de uso del cepillo eléctrico.

Al preguntar: "Comparando la facilidad de uso del cepillo eléctrico entregado, con los cepillos manuales que ha utilizado anteriormente, podría señalar que el cepillo eléctrico es". El $88 \%$ de los encuestados señaló que el cepillo eléctrico es más fácil de usar o mucho más fácil de usar que los cepillos manuales (más fácil de usar $57 \%$, mucho más fácil de usar $31 \%$ ).

Los resultados obtenidos frente a la pregunta: "Recomendaría el cepillo eléctrico entregado a otro paciente con frenillos?", se señalan en el siguiente gráfico (Fig. 3).

Los resultados obtenidos frente a la pregunta: "En general, en relación con él o los cepillos que usaba antes de formar parte de este estudio, considera que su nuevo cepillo es", se señalan en el siguiente (Fig. 4). Esto considerando que el $98 \%$ (41 de 42 participantes, utilizaba previamente cepillos manuales). 


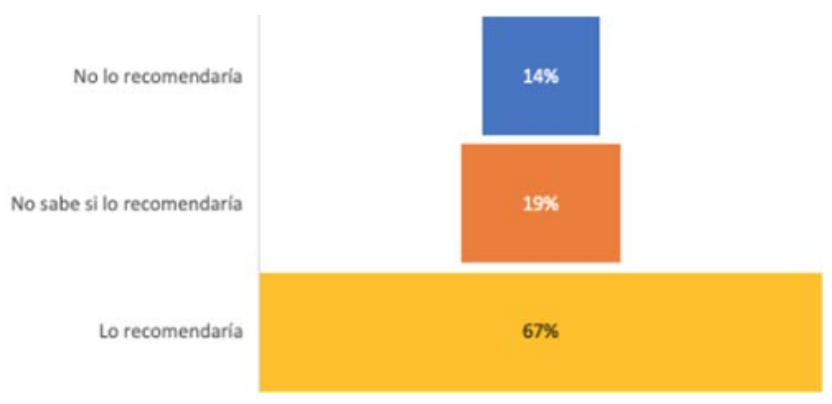

Fig. 3. Porcentajes de respuestas obtenidas en la encuesta en relación con la recomendación del uso de cepillo eléctrico en pacientes con aparatología fija.

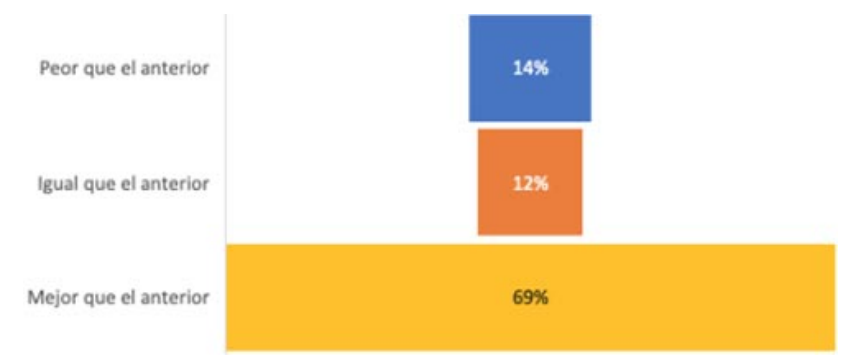

Fig. 4. Porcentajes de respuestas obtenidas en la encuesta en relación con la diferencia del cepillo eléctrico con su cepillo anterior.

En relación con los inconvenientes en el uso del cepillo eléctrico, sólo un $24 \%$ refirió presentarlos. Dentro de los problema más frecuentes mencionados se encuentran: dificultad de limpieza en zonas interproximales, dolor posterior a controles y daño en encías al principio de su uso, que se abren rápido las cerdas y que la vibración es muy fuerte.

Dentro de las características positivas que destacaron los encuestados se encuentran: su facilidad de uso, que se logra buena higiene, su comodidad, que limpia zonas difíciles. Las características que menos les gustaron fueron: la poca duración de la batería, el difícil acceso posterior y proximal y la vibración muy fuerte. Finalmente, dentro de las sugerencias a mejorar propuestas por los encuestados se encuentra considerar una mayor duración de la batería, reducir el tamaño del cepillo, y que la vibración sea más suave.

\section{DISCUSIÓN}

El presente estudio, randomizado y doble ciego, fue desarrollado en un plazo de 90 días, con el objetivo de determinar la eficacia en el control de pla- ca bacteriana y la percepción de los usuarios respecto de la comodidad y facilidad de uso del cepillo eléctrico Oral-B $\AA$ Professional Care 500 , en pacientes portadores de ortodoncia fija.

Al comparar los índices de higiene al inicio del estudio (TO), no se observaron diferencias estadísticamente significativas entre el grupo control y el experimental, lo que indica que los grupos a comparar comenzaron con similares condiciones de higiene.

Con respecto a los índices evaluados, se encontró una disminución estadísticamente significativa en el índice de O'Leary y el índice de placa en bracket a los 90 días de iniciado el estudio, tanto en pacientes que utilizaron cepillo eléctrico como en pacientes que utilizaron cepillos manuales, no encontrándose diferencias significativas en la magnitud de esta disminución entre ambos grupos.

El índice de Eastman, utilizado en este estudio, permite evaluar de una manera más simple el sangrado interproximal gingival, lo que da cuenta de la salud de las encías. En el estudio de Barendregt et al. (2002), se comprobó que no hubo una diferencia estadísticamente significativas entre el índice de sangrado del margen al sondaje con el índice de sangrado interproximal de Eastman, siendo ambos semejantes al evaluar el nivel de inflamación gingival (Barendregt et al.). Con respecto a este índice, se observó una disminución estadísticamente significativa en la cantidad de sitios con sangrado gingival, siendo tres veces mayor en el grupo que utilizó el cepillo eléctrico, sin embargo, esta diferencia resultó no ser significativa en el grupo que utilizó cepillos manuales. Estos resultados concuerdan con los obtenidos en Grender et al. (2020) y Deacon et al. (2010), en que se evalúo la reducción de sangrado gingival con el cepillo eléctrico Oral-B, disminuyendo tres veces más con el cepillo eléctrico. En estos estudio, se concluyó que el cepillo rotatorio-oscilante fue sustancialmente mejor en la reducción de placa y gingivitis que el cepillo manual (Deacon et al.; Grender et al.).

Existe limitada evidencia respecto del uso de cepillo eléctrico en pacientes con ortodoncia fija, sin embargo, en un estudio reciente de Erbe et al. (2019), se destaca que la mayor reducción de placa (36-38 $\%$ ) en este tipo de pacientes, se produjo con el uso de cepillo eléctrico. El desafío de la prescripción del cepillo puede ser aun mayor en pacientes adolescentes con ortodoncia fija. En los estudios de Erbe et al. y 
ARAYA-DÍAZ, P.; ABDALA, T. N.; YAGNAM, D. K.; PALOMINO, M. H.; PARADA, I. J. G.; PASTÉN, C. E. \& PIÑEIRO, B. S. Análisis comparativo de la eficacia, comodidad y seguridad del cepillo eléctrico oscilante-rotatorio Oral-B en pacientes portadores de ortodoncia fija: Un ensayo clínico aleatorizado. Int. J. Odontostomat. 15(3):765-772, 2021.

Nammi et al. (2019), se comprobó que el cepillo eléctrico fue más efectivo y seguro que la prescripción tradicional, favoreciendo la adherencia en las indicaciones de higiene dental es este tipo de pacientes.

En la encuesta de satisfacción, los pacientes que utilizaron el cepillo eléctrico lo consideraron más fácil de usar $(71 \%)$, más cómodo $(76 \%)$ y mejor que los cepillos manuales (69\%). De manera que un $67 \%$ de los encuestados lo recomendaría para pacientes portadores de aparatología fija.

Como factores a mejorar, se registró en la encuesta, el difícil acceso a las zonas interproximales al usar el cepillo eléctrico. Otros investigadores lograron mejorar esta complicación mediante la versión del cepillo con indicaciones bluetooth, prolongado el cepillado en las zonas de difícil acceso (Erbe et al.). Aún así, la aplicación del cepillo eléctrico permite disminuir la dependencia de la habilidad manual del operador para lograr la correcta higiene oral.

Se sugiere que posteriores investigaciones que incluyan el diagnóstico microbiológico, con recuento de especies bacterianas y que consideren la incidencia de lesiones de manchas blancas y su evolución durante el transcurso del tratamiento ortodóncico con el uso de cepillo eléctrico.

En el presente estudio se pudo comprobar que el cepillo eléctrico rotatorio-oscilante tiene ventajas sobre el cepillo manual para la higiene en pacientes con ortodoncia fija, bajo medidas objetivas, obteniéndose cambios mantenidos en el tiempo, elementos importantes a considerar al indicar la prescripción de cepillo dental en pacientes con mayores demandas de higiene.

\section{CONCLUSIONES}

Existe una disminución significativa en el índice de O'Leary y el índice de placa en bracket con el uso de cepillo eléctrico oscilante-rotatorio de Oral-B@ 500 y el uso de cepillos manuales en pacientes portadores de aparatología fija.

Se observó una disminución estadísticamente significativa en el índice de Eastman con el cepillo eléctrico oscilante-rotatorio de Oral-B@ 500, lo que da cuenta de un mejoramiento del estado de salud periodontal, no así con el uso de cepillos manuales.
El Cepillo eléctrico oscilante-rotatorio de Oral$B \subset 500$ fue considerado por la mayoría de los participantes, como un cepillo cómodo, fácil de usar, mejor que los cepillos manuales, y recomendable para pacientes con aparatología fija.

ARAYA-DÍAZ, P.; ABDALA, T. N.; YAGNAM, D. K.; PALOMINO, M. H.; PARADA, I. J. G.; PASTÉN, C. E. \& PIÑEIRO, B. S. Comparative analysis of the efficacy, comfort and safety of the Oral-B oscillating-rotary electric toothbrush in patients with fixed orthodontia: A randomized clinical trial. Int. J. Odontostomat., 15(3):765772, 2021.

ABSTRACT: Oral hygiene is essential in patients undergoing orthodontic treatment. Currently there is a wide offer of electric brushes for this purpose. The objective of this study is to determine the efficacy and comfort of the Oral-B® Professional Care 500 electric toothbrush in orthodontic wearers. A double-blind, single-center clinical trial was carried out, in which the sample was 90 patients, these were randomized into two groups: 45 patients with electric toothbrush treatment and 45 subjects with manual toothbrush treatment. The effectiveness was determined with the O'leary indexes, the bracket plate index and the Eastman index. For the evaluation of comfort, a survey was used. Statistical analysis was performed with the Shapiro-Wilks test and the Mann-Whitney $U$ test, in which a significant decrease was found in the O'leary index and bracket plate index in the group that used the electro-oscillating brush rotary and a greater decrease in the Eastman index was observed in the same group. In conclusion, the electric toothbrush is comfortable and easy to use, recommended for patients with fixed appliances.

KEY WORDS: orthodontics, toothbrushing, oral health, patient comfort, dental plaque index.

\section{REFERENCIAS BIBLIOGRÁFICAS}

Barendregt, D. S.; Timmerman, M. F.; van der Velden, U. \& van der Weijden, G. A. Comparison of the bleeding on marginal probing index and the Eastman interdental bleeding index as indicators of gingivitis. J. Clin. Periodontol., 29(3):195-200, 2002.

Bergamo, A. Z. N.; de Oliveira, K. M. H., Matsumoto, M. A. N.; do Nascimento, C., Romano, F. L.; da Silva, R. A. B.; da Silva, L. A. B. \& Nelson-Filho, P. Orthodontic appliances did not increase risk of dental caries and periodontal disease under preventive protocol. Angle Orthod., 89(1):25-32, 2019.

Deacon, S. A.; Glenny, A. M.; Deery, C.; Robinson, P. G.; Heanue, M.; Walmsley, A. D. \& Shaw, W. C. Different powered toothbrushes for plaque control and gingival health. Cochrane Database Syst. Rev., (12):CD004971, 2010. 
ARAYA-DÍAZ, P.; ABDALA, T. N.; YAGNAM, D. K.; PALOMINO, M. H.; PARADA, I. J. G.; PASTÉN, C. E. \& PIÑEIRO, B. S. Análisis comparativo de la eficacia, comodidad y seguridad del cepillo eléctrico oscilante-rotatorio Oral-B en pacientes portadores de ortodoncia fija: Un ensayo clínico aleatorizado. Int. J. Odontostomat. 15(3):765-772, 2021.

Dersot, J. M. Plaque control, a key element of successful orthodontics. Orthod. Fr., 81(1):33-9, 2010.

Erbe, C.; Klees, V.; Braunbeck, F.; Ferrari-Peron, P.; CcahuanaVasquez, R. A.; Timm, H.; Grender, J.; Cunningham, P.; Adam, R. \& Wehrbein, H. Comparative assessment of plaque removal and motivation between a manual toothbrush and an interactive power toothbrush in adolescents with fixed orthodontic appliances: A single-center, examiner-blind randomized controlled trial. Am. J. Orthod. Dentofacial Orthop., 155(4):46272, 2019

Grender, J.; Ram Goyal, C.; Qaqish, J. \& Adam, R. An 8-week randomized controlled trial comparing the effect of a novel oscillating-rotating toothbrush versus a manual toothbrush on plaque and gingivitis. Int. J. Dent., 70 Suppl. 1:S7-S15, 2020.

Heintze, S. D.; Jost-Brinkmann, P. G. \& Loundos, J. Effectiveness of three different types of electric toothbrushes compared with a manual technique in orthodontic patients. Am. J. Orthod. Dentofacial Orthop., 110(6):630-8, 1996.

Khoroushi, M. \& Kachuie, M. Prevention and treatment of white spot lesions in orthodontic patients. Contemp. Clin. Dent., 8(1):11-9, 2017.

Nammi, K.; Starke, E. M.; Ou, S. S.; Ward, M.; Jenkins, W.; Milleman, J. L. \& Milleman, K. R. The effects of use of a powered and a manual home oral hygiene regimen on plaque and gum health in an orthodontic population. J. Clin. Dent., 30 (Spec. No A):A18, 2019.

Sun, F.; Ahmed, A.; Wang, L.; Dong, M. \& Niu, W. Comparison of oral microbiota in orthodontic patients and healthy individuals. Microb. Pathog., 123:473-7, 2018.

Yaacob, M.; Worthington, H. V.; Deacon, S. A.; Deery, C.; Walmsley, A. D.; Robinson, P. G. \& Glenny, A. M. Powered versus manual toothbrushing for oral health. Cochrane Database Syst. Rev., 2014(6):CD002281, 2014.
Dirección para correspondencia:

Pamela Araya-Díaz

Facultad de Odontología

Universidad Andres Bello

calle Echaurren 237

Santiago

CHILE

E-mail: payleen@gmail.com 